\title{
Fast Near-Field Multi-Focusing of Antenna Arrays Including Element Coupling Using Neural Networks
}

\author{
Rafael G. Ayestarán, Senior Member, IEEE
}

(C) 2018 IEEE. Personal use of this material is permitted. Permission from IEEE must be obtained for all other uses, in any current or future media, including reprinting/republishing this material for advertising or promotional purposes, creating new collective works, for resale or redistribution to servers or lists, or reuse of any copyrighted

component of this work in other works.

IEEE link: https://ieeexplore.ieee.org/document/8365778

DOI: 10.1109/LAWP.2018.2840540

\begin{abstract}
A novel Near-Field Focusing approach based on the use of artificial Neural Networks is proposed. It is able to provide the set of weights or feeding values that must be applied to the elements of an array so that the global radiation/reception pattern is focused on one or more predefined positions in the near environment. Due to the use of a properly trained Neural Network, it is able to work fast enough for real time applications, such as wireless energy and information transfer where moving devices may require quick adaptation of the radiated field distribution, and hence of the weights applied to the array. Moreover, the training procedure using examples generated with a convenient electromagnetic analysis tool allows taking into account both the radiation pattern of the elements of the array and the coupling effects between them.
\end{abstract}

Index Terms-Near Field, focusing, multifocusing, neural networks, mutual coupling, wireless energy transfer.

\section{INTRODUCTION}

$\mathbf{N}$ EAR Field Focusing (NFF) has received increased attention in the last years as a technique for concentrating the energy radiated by an antenna on a predefined spot in the near-field region, reducing the energy wasted in other positions of the space where it is not required. This idea is key in novel paradigms such as Wireless Energy Transfer (WET), as well as other applications such as RFID [1], [2], medical hyperthermia [3], imaging, etc. [4]. In its more usual implementation, the Conjugate-Phase (CP) approach [1], [5], [6], it is extremely simple and robust and provides an excellent solution for problems that require concentrating the energy on one spot. However, among some minor drawbacks, an important limitation may be found: it is limited to one focal point. Some interesting beam steering methods have been proposed [7], but some applications may also require considering more than one focal point, concentrating the energy on an area or a combination of both points and areas as pointed out in [8], [9], where radiation nulls may be additionally requested.

Near Field Multifocusing (NFMF) is solved making use of optimization techniques aiming at the minimization of a

R. G. Ayestarán is with the Department of Electrical Engineering, Universidad de Oviedo, Gijón, Asturias, 33203 Spain, e-mail: rayestaran@uniovi.es.

This work was partially supported by the Spanish Ministerio de Economía, Industria y Competitividad under the projects TEC2014-54005-P (MIRIIEM) and TEC2017-86619-R (ARTEINE).

Manuscript submitted April 26, 2018, and revised May 10, 2018. proper cost function designed according to the specifications. Using this approach, more applications for NFF arise, specially simultaneous Wireless Energy and Information Transfer (WEIT) including the possibility of sending information to some devices while transferring energy to some other devices, so that the position of each device represents a focal point.

The price to pay for a increased focusing performance is time-cost, as far as the optimization process typically requires much more time that the $\mathrm{CP}$ approach. This could become an important limitation in applications such as WEIT where target devices might be moving, and hence the corresponding focal point and the time required to calculate the new weights to be applied to focus on the new positions might be excessive.

Neural Networks (NN) [10] have been used successfully in Far Field (FF) synthesis problems in antenna arrays [11], [12] showing an interesting performance, specially considering that once trained they are able to work without relevant timecost. They concentrate all the computing time in the previous training step, providing solutions almost in real time once trained. In this paper we propose the use of a trained NN to obtain the weights that must be applied to an array so that it is able to multifocus or to generate a Near Field (NF) distribution complying with the specifications and without an excessive delay that might be unacceptable for many applications.

\section{STATEMENT OF THE PROBLEM}

Let us consider a NF application where an antenna array with $N$ elements is used to focus on one or more points. The position of each element of the array is defined by the vector $\overrightarrow{r_{n}^{\prime}}=\left\{x_{n}^{\prime}, y_{n}^{\prime}, z_{n}^{\prime}\right\}, n=1 \ldots N$. Under the assumption of ideal isotropic elements without coupling or interaction between them, the field radiated by the $N$ elements of the array at any position $\vec{r}=\{x, y, z\}$ of the NF region is given by

$$
E(\vec{r})=\sum_{n=1}^{N} w_{n} \frac{e^{-j k\left|\vec{r}-\overrightarrow{r_{n}^{\prime}}\right|}}{\left|\vec{r}-\overrightarrow{r_{n}^{\prime}}\right|}
$$

where $w_{n} \in \mathbb{C}$ is the complex weight applied to the $n$-th element of the array, $k=2 \pi / \lambda$, and $\lambda$ is the wavelength. An optimization scheme is used in [9] improving [8] to focus simultaneously on a predefined set of $P$ points with positions given by $\vec{r}_{p}=\left\{x_{p}, y_{p}, z_{p}\right\}, p=1 \ldots P$, considering 
(1) as a sufficient approach for most applications. However, the radiation pattern of each element may be included by modifying the sum, so that it becomes

$$
E(\vec{r})=\sum_{n=1}^{N} w_{n} f_{n}\left(\vec{r}, \overrightarrow{r_{n}^{\prime}}\right) \frac{e^{-j k\left|\vec{r}-\vec{r}_{n}^{\prime}\right|}}{\left|\vec{r}-\overrightarrow{r_{n}^{\prime}}\right|}
$$

where $f_{n}\left(\vec{r}, \overrightarrow{r_{n}^{\prime}}\right)$ is a factor accounting for the radiation pattern of the $n$-th element, placed in $\overrightarrow{r_{n}^{\prime}}$, towards the direction defined by $\vec{r}$, and defining the field component represented by (2). A matrix-version of (2) is used to consider the field at different positions of the near-field region, so that

$$
\mathbf{e}=\mathbf{S} \cdot \mathbf{w}
$$

where $\mathbf{e}=\left[E\left(\overrightarrow{r_{1}}\right), E\left(\overrightarrow{r_{2}}\right) \ldots E\left(r_{M}\right)\right]^{T}$ is a column vector containing the values of the field at the $M$ considered positions, $\overrightarrow{r_{m}}, m=1 \ldots M$, of the near-field region; $\mathbf{w}=$ $\left[w_{1}, w_{2}, \ldots, w_{N}\right]^{T}$ is a vector with the weights applied to each element of the array; $\mathbf{S}$ is a matrix with elements $s_{n, m}=$ $f_{n}\left(\vec{r}_{m}, \overrightarrow{r_{n}^{\prime}}\right) \frac{e^{-j k\left|r_{m}-r_{n}^{\prime}\right|}}{\mid r_{m}^{\overrightarrow{r_{n}^{\prime}} \mid}}$; and $(.)^{T}$ stands for the transpose.

Additionally, a very simple and effective method to take into account coupling effects between the elements of the array consists on making use of a coupling matrix $\mathbf{A}$ [13], [14] with elements $a_{i, j}, i, j=1 \ldots N$ representing the influence of the $j$-th element in the effective weight applied to the $i$-th element. Equation (3) then may be rewritten as

$$
\mathbf{e}=\mathbf{S} \cdot \mathbf{A} \cdot \mathbf{w}
$$

Provided that the coupling matrix $\mathbf{A}$ is calculated accurately, (4) is a quite realistic representation of the behavior of the array in the near-field region. Some interesting methods for the calculation of the coupling matrix have been proposed, some of them leading to high accuracy results. In the results presented in this paper we have chosen the Method of Moments [15], able to create a quasi-realistic model accounting for the real properties of the radiating structure without any need of measured data. Once the coupling matrix is calculated, (4) may be used in [9] to relate the weights applied to the array and the corresponding NF distribution, instead of the formulation actually used in [9]. However, time requirements remain high due to the iterative nature of optimization tools.

\section{NeUral Networks as a FASt NeAR FIELD FOCUSING METHOD}

Neural Networks [10] are the most popular Machine Learning tool in the scientific literature due in part to their ease of use and their excellent results in solving learning-by-examples problems. They have been used with success in a large number of applications, including the synthesis of FF patterns in antenna arrays [16], [11], [12]. Among the interesting capabilities of NNs, they displace the computational time and cost to a previous training stage when the NN learns the behavior of the system to be modeled, e.g. a given antenna array, before being used for operation, and then, once trained, they operate extremely fast and with a very reduced computational cost.

In the most general case, NNs are designed to model a function that relates inputs and outputs through the use of combinations of neurons, that basically are linear combinators of their inputs followed by an activation function. This activation function is usually chosen to make use of the principle of Universal Approximation [10], that states that any function may be approximated with arbitrary accuracy provided that certain activation functions are chosen (typically gaussian or sigmoid functions) and a large enough number of neurons are used. For synthesis purposes, a two-layer NN with gaussian functions in the first layer and linear functions in the output layer has been shown to be highly effective [12].

The key of the accurate modeling of the problem by the NN is the training stage. A set of inputs and their corresponding outputs must be presented to the $\mathrm{NN}$ so that it is able to learn the relationship between both. For the NFF problem we expect the NN to receive the target NF distribution and to output the set of weights to be applied to the array, so a set of training patterns must be constructed. To do so, (4) may be used generating a set of $P$ random weight vectors, $\{\mathbf{w}\}_{r}, r=1 \ldots P$, and obtaining the corresponding set of field samples vectors $\{\mathbf{e}\}_{r}$. It is important to notice that most NN implementations are unable to deal with complex values, and both field sample and weight values must be decomposed into their real and imaginary parts, being the actual training patterns $\{\tilde{\mathbf{e}}, \tilde{\mathbf{w}}\}_{r}$ with $\tilde{\mathbf{e}}=\left[\Re\left(e^{T}\right) \Im\left(e^{T}\right)\right]^{T}$ and $\tilde{\mathbf{w}} w=\left[\Re\left(w^{T}\right) \Im\left(w^{T}\right)\right]^{T}$ being extended versions of $\mathbf{e}$ and $\mathbf{w}$ obtained by stacking their real and imaginary parts. These patterns are analyzed by the NN until it is properly trained, and it is able to perform synthesis tasks.

The method presented in [9] defines a target field distribution consisting on a unitary value for the samples corresponding to the spots where focus is required, and null values for any other sample. This is a very flexible procedure that allows specifying multiple spots, arbitrary volumes, etc. Although the resulting target distribution is obviously unreachable, it has been found to be quite effective for NFF purposes. In the NN-based approach proposed in this paper the same design procedure will be used for specifying a NF distribution according to the NFF requirements.

\section{RESUlts}

In order to test the proposed method, a planar antenna array with $12 \times 12$ elements $(N=144)$ uniformly distributed and centered in the plane $z=0$, with separation $0.6 \lambda$ between them, is considered. The chosen individual radiating elements are hemispherical dielectric resonator antennas (DRA) [17], interesting due to their low losses but with relevant mutual coupling effects when included in an array. For each element, the radius of the hemisphere is $12.5 \mathrm{~mm}$, and the relative permitivity is $\epsilon_{r}=9.8$. The DRA is fed by a metallic pin with radius $0.63 \mathrm{~mm}$, height $6.5 \mathrm{~mm}$ and offset $6.5 \mathrm{~mm}$. The working frequency is $3.6 \mathrm{GHz}$ in order to excite the $T E_{11}$ mode. The range of variation of $S_{11}$ for the elements is between -10.17 and $-13.72 \mathrm{~dB}$, being the maximum of the rest of $S$ parameters $-12.35 \mathrm{~dB}$, so the coupling effects are relevant and have to be accounted for. An infinite ground plane is considered in the simulation. MoM was used to obtain the coupling matrix by calculating the currents induced at each 
element by the other elements when they are excited with a voltage source [14], [13].

The near-field region around the antenna has been limited to $x \in[-7 \lambda, 7 \lambda], y \in[-5 \lambda, 5 \lambda]$ and $z \in[0.5 \lambda, 10 \lambda]$, sampled with a period $\lambda / 2$ what leads to 12180 samples of the field distribution. The NN consists on two layers, the first one with 1200 neurons with gaussian activation function, and the output layer using linear activation function and 288 neurons (one for each output, being each output the real or imaginary part of a weight). A set of 5000 training patterns have been used each one consisting on a set of 144 complex weights and using (4) to calculate the corresponding NF distributions. The algorithm used for training procedure was the standard Least Squares for the lineal layer with k-means and pseudoinversion for the gaussian layer [10].

Both the optimization method and the trained $\mathrm{NN}$ are used to calculate the weights that must be applied to the array in order to obtain a simultaneous focus on three focal points at $\{2 \lambda, 0,7 \lambda\},\{-\lambda, 0,4 \lambda\}$ and $\{-3 \lambda, 0,4 \lambda\}$. The weights obtained by each technique have been applied to (4) to calculate the corresponding radiated field, that can be calculated for any required position independently of the grid of the environment used to train the $\mathrm{NN}$ or to define the target distribution. Figure 1 shows the NF power density distribution in the plane $y=0$ where all the focal points have been specified. The optimization method in [9] only generates two $-3 \mathrm{~dB}$ focal spots; the third spot does not reach that level, and one of the focal points lays out of the corresponding spot. In opposition, the neural network is able to generate $-3 \mathrm{~dB}$ focal spots containing the three focal points. Moreover, the optimization has spent $102.28 \mathrm{~s}$, too much for most WET or WEIT applications, while the neural network has spent $0.3 \mathrm{~s}$, suitable for most cases. The calculations have been carried out in a conventional PC with an Intel Core 2 Duo CPU, 2 $\mathrm{GHz}$ and 4 GB RAM, using Octave as programming tool, and averaging 20 simulations for both methods. These results agree with the complexity of both methods, provided that the NN has a complexity of $\mathcal{O}(N)$ for $N$ elements in the array [18], while the Levenberg-Marquardt algorith used in [9] has a complexity $\mathcal{O}\left(N^{3}\right)$ per iteration [19].

The positions of the resulting maximum points also differ from one method to the other. Figure 1 shows the specified focal points along with the obtained spots and maximum points. The optimization scheme is reaching maxima on $\{1.8 \lambda, 0,5.1 \lambda\},\{-0.9 \lambda, 0,2.8 \lambda\}$ and $\{-2.8 \lambda, 0,3.1 \lambda\}$, what represents an average distance $1.34 \lambda$ between the focal points and the maximum points, while the neural network is getting maxima on $\{1.8 \lambda, 0,5.8 \lambda\},\{-1 \lambda, 0,3.3 \lambda\}$ and $\{-2.8 \lambda, 0,3.7 \lambda\}$ what represents a lower average distance $0.75 \lambda$ between the focal and maximum points. This improved focusing accuracy is actually due to the use of (4) including the radiating properties of the elements of the array and the coupling effects in the formulation of the problem. In fact, if (4) is used as formulation in the optimization method instead of the non-coupling array used in [9], both methods converge to the same solution being the advantages of the neural network reduced to its fast operation once trained. In this case, no differences may be appreciated in the field

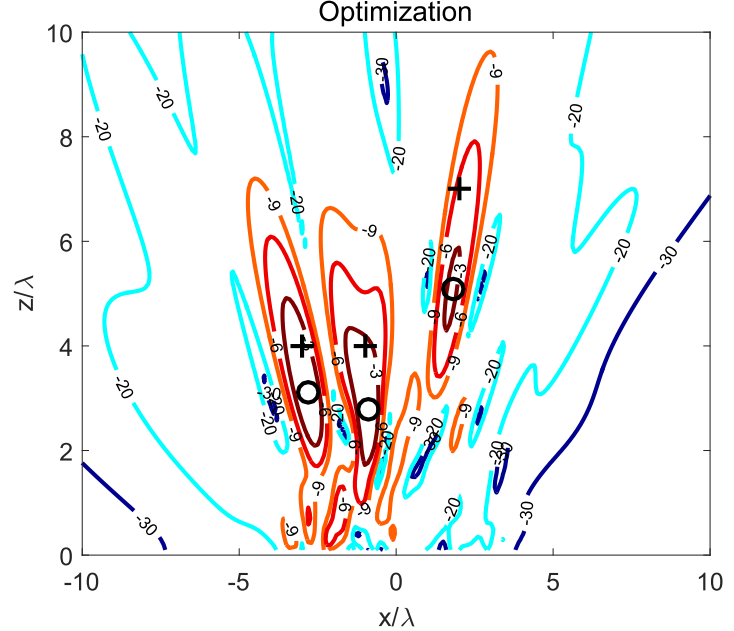

(a)

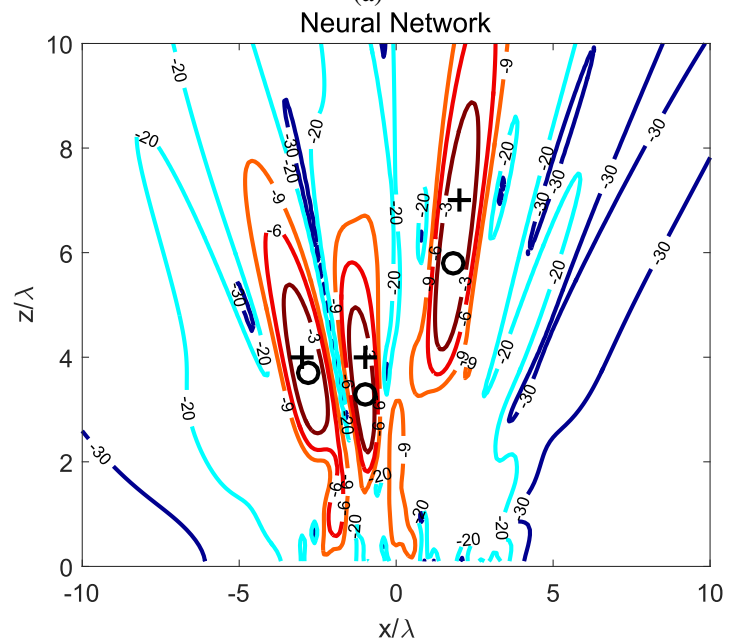

(b)

Fig. 1: Example \#1. Normalized Near Field power density at $y=0$ for the optimization scheme in [9] (a) and the neural network (b). The symbols + and $\circ$ represent the focal and synthesized maximum points respectively.

distribution generated by the array when using both methods but the computation time is still high using the optimization tool.

The use of a model including coupling effects provides an improved efficiency in power transfer that can be estimated by measuring the power density in the focal points when the transmitted power is the same in both methods. The gain obtained in those points $G_{\epsilon}$ may be estimated as

$$
G_{\epsilon}=\frac{P_{D}^{C}}{P_{D}^{I}}
$$

where $P_{D}^{C}$ is the power density at a focal spot considering coupling effects and $P_{D}^{I}$ is the power density at the same spot under ideal conditions (no coupling is considered). For the results obtained in this experiment, the gain at each focal point has been found to be 2.34, 1.06 and 1 respectively. Obviously, if coupling effects are taken into account in the optimization tool both methods reach the same solution and hence the same gain. Table I summarizes the axis lenght for the 


\begin{tabular}{|c|c|c|c|}
\hline Focal point & $\{2 \lambda, 0,7 \lambda\}$ & $\{-\lambda, 0,4 \lambda\}$ & $\{-3 \lambda, 0,4 \lambda\}$ \\
\hline Axis length Opt & $1.64 \lambda, 0.28 \lambda$ & $2.57 \lambda, 0.98 \lambda$ & $2.97 \lambda, 0.76 \lambda$ \\
\hline$\rho$ Opt (dB) & 7.75 & 2.49 & 1.62 \\
\hline Axis length NN & $4.85 \lambda, 1.22 \lambda$ & $2.74 \lambda, 0.84 \lambda$ & $2.97 \lambda, 0.94 \lambda$ \\
\hline$\rho$ NN (dB) & 0.64 & 1.08 & 0.55 \\
\hline
\end{tabular}

TABLE I: Example \#1. Results using optimization and NN. The power density ratios are obtained using the same radiated power.

$-3 \mathrm{~dB}$ focal spots, and the ratio $\rho$ between the power density at the maximum points and the power density at the focal points.

In a second example, only one focal point has been considered so that a comparison between the proposed neural network and the CP method may be achieved. The CP approach only can deal with one focal point, but it is very fast. The main point for comparison is the ability of the proposed neural method to account for the coupling effects. The focal point has been placed in the position $\{2 \lambda, 0,7 \lambda\}$. The same array considered for the first example has been used here, as well as the same NN trained for that array. The normalized NF power density obtained with both methods is represented in Fig. 2. It may be noticed that a better fit is obtained using the neural method, whose actual synthesized maximum point is at $\{1.8 \lambda, 0,5.9 \lambda\}$ while the maximum point synthesized by the $\mathrm{CP}$ method is at $\{1.8 \lambda, 0,5.5 \lambda\}$. The use of the neural tool accounting for propagation losses and coupling effects has reduced the focal shift from $1.51 \lambda$ to $1.11 \lambda$. The difference between the power in the maximum point and the focal point is $1.29 \mathrm{~dB}$ using the $\mathrm{CP}$ method and $0.57 \mathrm{~dB}$ using the NN. The spot axis lengths in the $\mathrm{CP}$ method are $4.59 \lambda$ and $0.9 \lambda$ while the lengths in the NN method are $4.78 \lambda$ and $\lambda$. The gain $G_{\epsilon}$ has also been evaluated, resulting a value of only 1.1 for this case as both methods get spots including the focal points. It is interesting (but not surprising) to notice that the CP method has been found to be faster than the NN, with an average time of 0.1 s over 20 simulations (complexity $\mathcal{O}(N)$ ) while the NN has required an average time of $0.27 \mathrm{~s}$. The obvious conclusion is that the CP approach is still very convenient in cases where couplings are not relevant and only one focal point is specified but a very fast response is required, while the NN approach is very competitive in the case of multifocus with relevant couplings that must be considered in a fast system.

A third comparison may be performed by considering the NN method used for multifocus presented in Fig. $1 \mathrm{~b}$ and the $\mathrm{CP}$ approach presented in Fig. 2a, as both examples share one focal point. Even though both methods are targeting different specifications, the results obtained in the previous experiments for the common focal point may be compared. It is interesting to point out how the focal shift reduction by the NN leads to a lower ratio between the power level at the maximum point and at the focal point, $0.64 \mathrm{~dB}$, while the $\mathrm{CP}$ was getting $1.29 \mathrm{~dB}$, even when the array focusing capabilities are divided into three spots. Recall that the $-3 \mathrm{~dB}$ spot axis lengths were $\{4.85 \lambda, 1.22 \lambda\}$ for the $\mathrm{NN}$ and $\{4.59 \lambda, 0.9 \lambda\}$ for the CP.

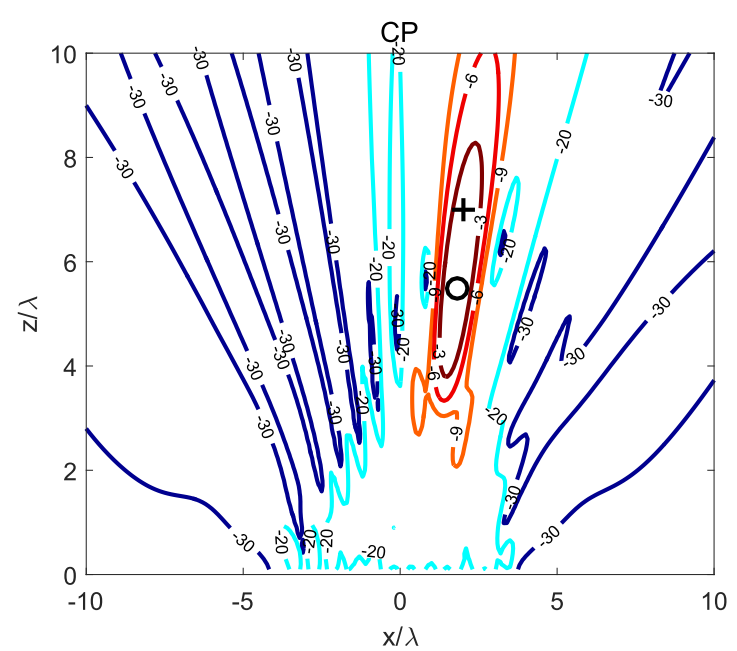

(a)

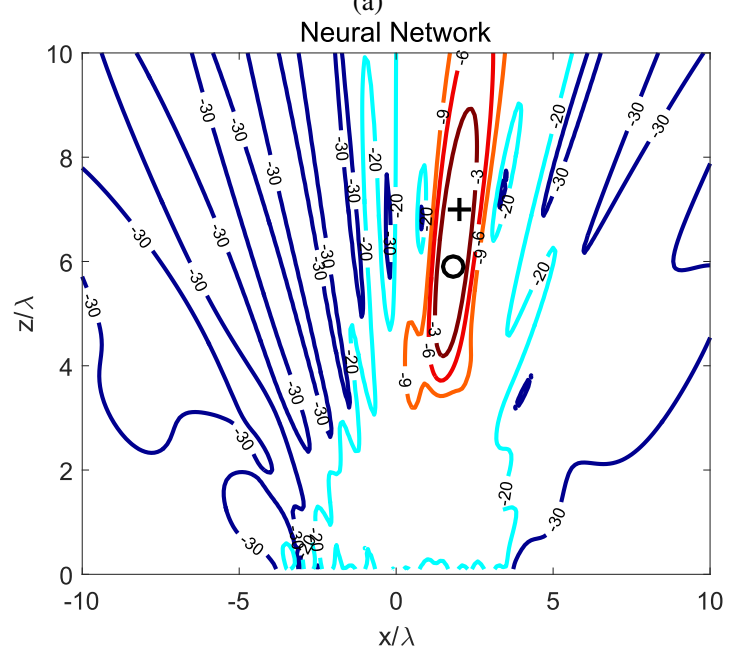

(b)

Fig. 2: Example \#2. Normalized Near Field power density at $y=0$ for the CP method (a) and the neural network (b). The symbols + and $\circ$ represent the focal and synthesized maximum points respectively.

\section{CONClusion}

A novel approach to Near Field Focusing based on the use of a properly trained Neural Network has been presented. It is intended as a fast synthesis method able to operate in real situations where real-time calculations are required. Although the most conventional approach to NFF, Conjugate-Phase, is usually very fast, it cannot deal with multiple specifications, while an optimization approach, able to account for multiple focal points at the same time, is not fast enough to be used in real scenarios where focusing might be specified on moving devices defined by moving focal points. The neural approach takes advantage of its properties to show the benefits of both previous techniques. Additionally, it is able to account for real properties of the array based on a formulation that might be also used in optimization schemes and that includes both the radiation pattern of each element of the array and coupling effects in the structure. As a limitation to be addressed in future developments, most implementations of NNs are not able to handle additional constraints in the solutions, what 
means that they are not able to provide phase-only solutions for the weights to be applied to the array as optimization tools have been shown to be. Anyway, NNs represent an interesting step forwards in the way to implementing real wireless energy transfer systems on applications with different devices involved placed in the near-field regions, even when some of them are moving.

\section{REFERENCES}

[1] A. Buffi, A. A. Serra, P. Nepa, H. T. Chou, and G. Manara, "A focused planar microstrip array for $2.4 \mathrm{ghz}$ rfid readers," IEEE Transactions on Antennas and Propagation, vol. 58, no. 5, pp. 1536-1544, May 2010.

[2] H. T. Chou, T. M. Hung, N. N. Wang, H. H. Chou, C. Tung, and P. Nepa, "Design of a near-field focused reflectarray antenna for $2.4 \mathrm{ghz}$ rfid reader applications," IEEE Transactions on Antennas and Propagation, vol. 59, no. 3, pp. 1013-1018, March 2011.

[3] W. Gee, S. W. Lee, N. K. Bong, C. A. Cain, R. Mittra, and R. L. Magin, "Focused array hyperthermia applicator: Theory and experiment," IEEE Transactions on Biomedical Engineering, vol. BME-31, no. 1, pp. 3846, Jan 1984.

[4] A. Buffi, P. Nepa, and G. Manara, "Design criteria for near-field-focused planar arrays," IEEE Antennas and Propagation Magazine, vol. 54, no. 1, pp. 40-50, Feb 2012.

[5] P. Nepa and A. Buffi, "Near-field-focused microwave antennas: Nearfield shaping and implementation." IEEE Antennas and Propagation Magazine, vol. 59, no. 3, pp. 42-53, June 2017.

[6] P. Nepa, A. Buffi, A. Michel, and G. Manara, "Technologies for nearfield focused microwave antennas," International Journal of Antennas and Propagation, vol. 2017, p. 17 pages, 2017.

[7] H. T. Chou, M. Y. Lee, and C. T. Yu, "Subsystem of phased array antennas with adaptive beam steering in the near-field rfid applications," IEEE Antennas and Wireless Propagation Letters, vol. 14, pp. 1746$1749,2015$.

[8] J. Alvarez, R. G. Ayestaran, and F. Las-Heras, "Design of antenna arrays for near-field focusing requirements using optimisation," Electronics Letters, vol. 48, no. 21, pp. 1323-1325, October 2012.

[9] J. Alvarez, R. G. Ayestaran, G. Leon, L. F. Herran, A. Arboleya, J. A. Lopez-Fernandez, and F. Las-Heras, "Near field multifocusing on antenna arrays via non-convex optimisation," IET Microwaves, Antennas Propagation, vol. 8, no. 10, pp. 754-764, July 2014.

[10] S. Haykin, Neural Networks : A Comprehensive Foundation. Mcmillan College Publishing Company Inc., 1994.

[11] R. G. Ayestaran and F. L. Heras, "Neural networks and equivalent source reconstruction for real antenna array synthesis," Electronics Letters, vol. 39, no. 13, pp. 956-958, June 2003.

[12] R. G. Ayestaran, F. Las-Heras, and L. F. Herran, "Neural modeling of mutual coupling for antenna array synthesis," IEEE Transactions on Antennas and Propagation, vol. 55, no. 3, pp. 832-840, March 2007.

[13] J. Alvarez, R. G. Ayestaran, J. Laviada, and F. Las-Heras, "Support vector regression for near-field multifocused antenna arrays considering mutual coupling," International Journal of Numerical Modelling: Electronic Networks, Devices and Fields, vol. 29, no. 2, pp. 146-156, 2016.

[14] R. G. Ayestaran, J. Laviada, and F. Las-Heras, "Realistic antenna array synthesis in complex environments using a mom-svr approach," Journal of Electromagnetic Waves and Applications, vol. 23, no. 1, pp. 97-108, 2009.

[15] R. F. Harrington, Field Computation by Moment Methods. Wiley-IEEE Press, 1993.

[16] A. Rawat, R. Yadav, and S. Shrivastava, "Neural network applications in smart antenna arrays: A review," AEU - International Journal of Electronics and Communications, vol. 66, no. 11, pp. 903 - 912, 2012.

[17] M. W. McAllister and S. A. Long, "Resonant hemispherical dielectric antenna," Electronics Letters, vol. 20, no. 16, pp. 657-659, August 1984

[18] W. T. C. W.-T. Chen and K. R. H. K.-R. Hsieh, "A neural sorting network with o(1) time complexity," in 1990 IJCNN International Joint Conference on Neural Networks, June 1990, pp. 87-95 vol.1.

[19] G. Dartmann, E. Zandi, and G. Ascheid, "A modified levenbergmarquardt method for the bidirectional relay channel," IEEE Transactions on Vehicular Technology, vol. 63, no. 8, pp. 4096-4101, Oct 2014. 